\title{
Norois
}

Environnement, aménagement, société

\section{Transversalité de l'Observatoire photographique du paysage de la LGV Bretagne-Pays de la Loire}

The tranversality of the brittany-Pays de la loire HSL photographic landscape observatory

\section{Caroline Guittet et Anne-Laure Bethe}

\section{OpenEdition}

\section{Journals}

Édition électronique

URL : http://journals.openedition.org/norois/6967

DOI : 10.4000/norois.6967

ISSN : $1760-8546$

\section{Éditeur}

Presses universitaires de Rennes

\section{Édition imprimée}

Date de publication : 5 novembre 2018

Pagination : 67-90

ISBN : 978-2-7535-7627-8

ISSN : 0029-182X

\section{Référence électronique}

Caroline Guittet et Anne-Laure Bethe, «Transversalité de l'Observatoire photographique du paysage de la LGV Bretagne-Pays de la Loire », Norois [En ligne], 248 | 2018, mis en ligne le 05 novembre 2020, consulté le 05 janvier 2021. URL : http://journals.openedition.org/norois/6967 ; DOI : https://doi.org/ 10.4000 /norois. 6967 


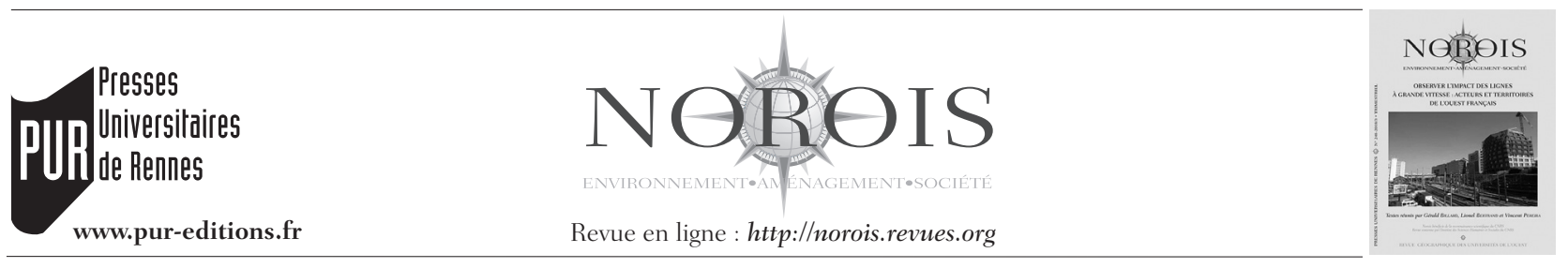

\title{
Transversalité de l'Observatoire photographique du paysage de la LGV Bretagne-Pays de la Loire
}

\author{
The Tranversality of the Brittany-Pays de la Loire HSL Photographic Landscape Observatory
}

\author{
Caroline Guittet ${ }^{\mathrm{a}}$ et Anne-Laure Bethe ${ }^{\mathrm{b}}$
}

\footnotetext{
a ESO-Rennes, UMR CNRS 6590 ESO, université Rennes 2 - Place du recteur-Le-Moal, CS24307, 35043 RENNES cedex, France. (caroline.guittet@univ-rennes2.fr)

b Observatoire d'Eiffage Rail Express - ZA la Servinière, 53940 SAint-Berthevin, France. (Anne-Laure.Bethe@ eiffage.com)
}

Résumé : L’implantation de la LGV Bretagne-Pays de la Loire a des impacts directs et indirects sur les territoires engendrant des transformations paysagères. Dans le but de les observer, Eiffage Rail Express a mis en place une veille paysagère via son Observatoire photographique du paysage (OPP). Cet outil est transversal à l'Observatoire environnemental et participe à l'enrichissement de la connaissance paysagère à l'échelle de la Bretagne. Cette contribution vise à montrer comment la démarche de l'OPP permet une analyse des dynamiques paysagères donnant à voir et à comprendre les processus de cicatrisation des paysages.

Abstract: The creation of the Brittany-Pays de la Loire high-speed rail line has direct and indirect impacts on the territories through which it passes, leading to changes in landscape dynamics. Eiffage Rail Expess has set up a Photographic Landscape Observatory in order to monitor these changes. The Photographic Landscape Observatory is a transverse tool of the Environmental Observatory, and a tool that helps to enrich knowledge at a regional level in Britanny. This paper aims to show how this approach makes it possible to analyze landscape dynamics with a view to highlighting and explaining landscape scarring processes.

Mots clés : observatoire - représentation paysagère - transition paysagère - acteurs locaux - politique d'aménagement - train à grande vitesse

Keywords: observatory - landscape representations - landscape transition - local actors - planning policy - high speed train

\section{INTRODUCTION}

L'implantation des grandes infrastructures de transport perturbe les rythmes des paysages qui sont en projet. Cette "phase de synchronie », cette rupture dans la permanence des formes est observable dans la matérialité visible des paysages mais aussi dans les pratiques et les usages des populations qui sont soumis à la modification des lieux (Chouquer, 2000). La nécessaire rectitude des Lignes à Grande Vitesse (LGV) bouscule les formes paysagères - remblais monumentaux, percement des collines et des forêts, etc. - pour, in fine, modifier les caractéristiques du territoire. Dans le langage commun, on parle de « cicatrisation ». Les paysages sont soumis à certains moments à de fortes perturbations 
d'ordre géologique (tectonique des plaques, etc.), historique (Grande Guerre, etc.) ou liées aux activités humaines (autoroutes, exploitation minière, etc.) créant ainsi une rupture paysagère. La cicatrisation désigne alors les processus mis en œuvre pour absorber les perturbations (Bailly, 2013). Ce néologisme nous indique que les paysages sont résilients : par les processus naturels et/ou humains, l'intégration des perturbations s'effectue de « manière souvent positive, créatrice, grâce à de multiples changements et réajustements " sans pour autant modifier en profondeur les paysages (Aschan-Leygonie, 2000, p. 65).

Dans le cadre de l'Observatoire socio-économique et environnemental de la LGV et plus spécifiquement dans le volet environnemental, Eiffage Rail Express (ERE) s'est attaché à comprendre ces processus de transformation via la mise en place d'un Observatoire photographique du paysage (OPP). Il s'agit de photographier à des intervalles de temps réguliers les mêmes paysages afin d'évaluer les impacts directs et indirects de la LGV puis d'observer leur cicatrisation. La démarche est originale en deux points ${ }^{1}$. D'une part, rares sont les entreprises privées qui conçoivent cet outil, il est surtout employé par des institutions publiques (Parc Naturel Régional, syndicat mixte, agglomération, etc.) en partenariat avec l'État dans le cadre de l'Observatoire Photographique National du Paysage (OPNP) ou de manière indépendante. D'autre part, la commande passée avec SNCF Réseau ne prescrivait pas un OPP dans une optique d'évaluation approfondie des mutations paysagères.

La notion de "transversalité » semble qualifier l'OPP de la LGV : (i) « qui coupe en travers un axe longitudinal »; (ii) qui relie deux voies de circulation parallèles ou divergentes à partir d'un centre $^{2} »$. L'objectif de l'article est de questionner l'OPP en tant qu'outil transversal pour appréhender les différentes problématiques relatives à l'implan-

1. Voici quelques exemples d'OPP dans le grand ouest de la France : l'OPP de l'Orne, passage de l'autoroute A26 porté par le Conseil d'Architecture, d'Urbanisme et de l'Environnement de l'Orne (CAUE); l'OPP des sites des Pays-de-la-Loire porté par la Direction Régionale de l'Environnement, de l'Aménagement et du Logement Pays-de-la-Loire; l'OPP du pays de Saint-Brieuc porté par le Syndicat mixte du pays de Saint-Brieuc; l'OPP des friches urbaines de Blois porté par l'agglomération de Blois. En comparaison avec l'OPP de la LGV Bretagne - Pays-de-la-Loire, tous sont portés par une institution publique. Ces OPP sont conçus de manière intrinsèque dans l'optique de réaliser un suivi photographique.

2. Définitions issues du Centre National de Ressources Textuelles et Lexicales. tation de la LGV et à la réorganisation territoriale. En d'autres termes, l'OPP relie-t-il les différentes problématiques dans une optique de créer une vision d'ensemble des impacts de la LGV? Aussi, dans quelle mesure l'OPP permet-il une analyse des dynamiques paysagères?

À partir d'un état de l'art des OPP en France et à l'international, la première partie de cette contribution a pour but de contextualiser l'Observatoire photographique du paysage de la LGV Bretagne-Pays de la Loire dans les expertises photographiques des grandes infrastructures de transport. Le second volet est consacré à expliquer et à interroger les spécificités du suivi photographique de la Ligne à Grande Vitesse, des spécificités qui sont adaptées à l'échelle et aux enjeux du projet. La dernière partie vise à questionner la notion de transversalité de l'OPP et à discuter des apports et des limites de l'outil dans l'analyse des dynamiques paysagères.

\section{L'EXPERTISE PHOTOGRAPHIQUE DES INFRASTRUCTURES DE TRANSPORT}

Eiffage Rail Express met en place l'Observatoire photographique du paysage de la LGV Bretagne-Pays de la Loire en 2014, deux ans après le lancement de l'Observatoire socio-économique et environnemental. Cet OPP s'intègre dans une démarche nationale qui perdure depuis une trentaine d'années. Dès le dernier quart du XIX ${ }^{\mathrm{e}}$ siècle, les contours méthodologiques se dessinent.

\section{Plus d'un siècle d'expertise photographique}

Le suivi photographique des travaux est une pratique déjà ancienne. Les services de Restauration des Terrains en Montagne (RTM) constituent à partir de 1886 et jusqu'en 1940 un fonds photographique composé de prises de vue reconduites de manière régulière. Ces services ont pour mission « de lutter contre l'érosion et le déboisement accéléré des montagnes qui provoquent des inondations dans les plaines » (Lebart, 1997, p. 2). Pour chaque photographie, les agents de la RTM marquent le lieu de prise de vue. Ils renseignent les coordonnées géographiques, la date et l'azimut, ils légendent aussi la photographie tout comme les OPP actuels. Les ingénieurs forestiers peuvent se mettre en scène 
pour donner une notion d'échelle géographique. Ils sont par ailleurs formés à la pratique de la photographie. En comparaison, les Observatoires contemporains sont conçus soit par des professionnels de la photographie soit par des chargés de mission n'ayant, en règle générale, aucune formation. Cette question est matière à débat et interroge le statut des photographies entre œuvre d'art, image d'amateur ou photographie documentée.

Dans le cadre de la RTM, le suivi photographique a un triple objectif selon Juliette Carré et Jean-Paul Métailié (2008) : (i) la photographie répétée permet de documenter le terrain et appréhender les évolutions forestières; (ii) elle est pédagogique pour montrer aux responsables les dégradations des montagnes; (iii) elle est politique, elle prouve l'efficacité des actions des ingénieurs.

À la fois objet de propagande et preuve de la gestion de la nature, la photographie répétée pour l'observation de la forêt sera pratiquée par la suite en Italie, en Suisse et en Autriche (Carré et Métailié, 2008). À proprement parler des grandes infrastructures de transport, l'une des premières expertises photographiques serait sans doute lors de la construction des portions de la Lincoln Highway ${ }^{3}$ dans l'état de l'Utah avec des prises de vue toutes les une mile en 1913 et en $1919^{4}$.

C'est beaucoup plus tard, en 1991, que le dispositif des Observatoires photographiques du paysage se formalise sous l'impulsion du ministère de l'Environnement. Officialisé en 1994, l'Observatoire photographique national du paysage a pour dessein de représenter les principales problématiques paysagères au niveau national. Le Bureau des paysages ${ }^{5}$ accompagne les institutions locales volontaires qui ont des missions d'aménagement du territoire et du paysage pour concevoir un itinéraire photographique. Ce dernier est « un parcours virtuel dans un territoire, qui rend compte des préoccupations des maîtres d'ouvrage en matière de paysage » (MEDDAT, 2008, p. 60). Pour le mettre en œuvre, une méthode spécifique est requise (figure 1).

Un OPP est le fruit des réflexions d'un comité de pilotage composé d'élus locaux, de services déconcentrés de l'État, de professionnels et d'asso-

3. Route transcontinentale reliant les côtes Est et Ouest des États-Unis.

4. À ce propos : [www.dri.educ/linciln-hihway].

5. Le Bureau des paysages est l'organisme qui traite des questions de paysage au ministère chargé de l'Environnement.

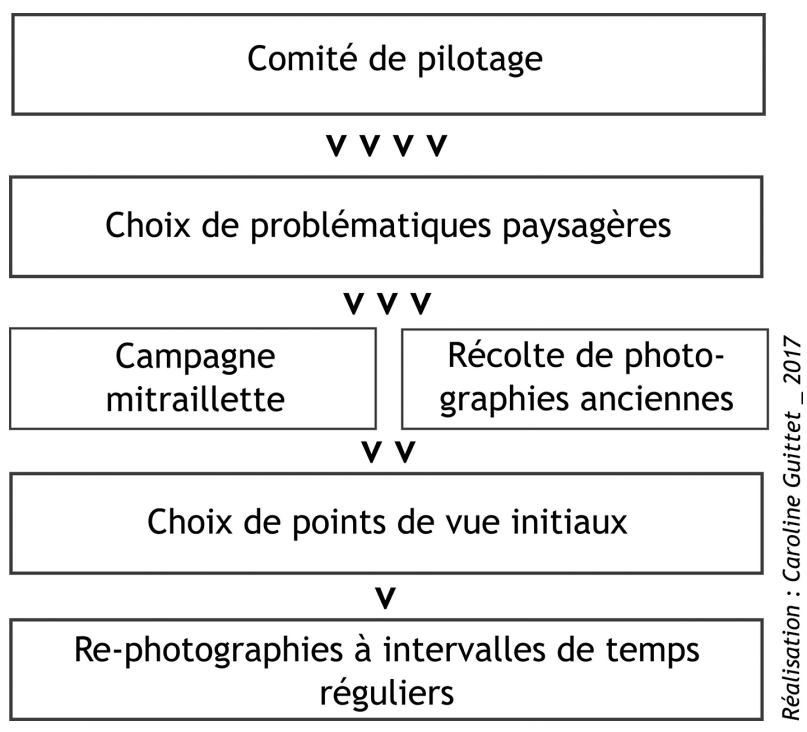

Figure 1 : Méthode de conception d'un Observatoire photographiques du paysage tory

Method for creating a Photographic Landscape Observa-

ciations, composé en définitive de la gouvernance territoriale. Le pilotage à l'échelle locale permet de saisir les dynamiques paysagères en œuvre sur le territoire puisqu'elles sont connues des acteurs. La concertation est l'occasion de développer une culture commune des problématiques paysagères qui sont à traiter par cette même gouvernance. La limite principale de cette réflexion « de l'intérieur » est le manque de recul sur certaines dynamiques qui sont encore peu visibles, qui n'ont pas encore potentiellement alarmés les acteurs locaux ou qui sont devenues invisibles au quotidien. Le regard d'un expert extérieur au territoire peut contrecarrer ce manque de recul.

Après avoir sélectionné les problématiques paysagères actuelles et à venir sur le territoire donné, le comité de pilotage mandate un photographe (professionnel ou non) pour effectuer une campagne « mitraillette », c'est-à-dire des prises de vue en grand nombre répondant aux problématiques définies en amont. Cette première campagne peut être menée en parallèle avec la récolte de photographies anciennes. Le comité de pilotage choisit ainsi, dans ce corpus, un ensemble de points de vue initiaux qui sera photographié à des intervalles de temps réguliers selon un protocole de reconduction rigoureux. Cet ensemble, l'itinéraire photographique, est composé d'un nombre de points de vue initiaux limité afin d'en assurer la reconduction (entre 20 et 80). 
Les temps de reconduction sont déterminés selon les dynamiques paysagères suivies (tous les ans, tous les cinq ans, tous les dix ans) par le comité de pilotage. Dans la pratique, les intervalles sont relativement courts afin de disposer d'une chronique photographique précise (reconduction annuelle ou tous les deux ans).

Ainsi les Observatoires ont pour but de constituer des archives de photographies répétées à partir d'une démarche singulière (comité de pilotage, sélection de problématiques paysagères, photographies répétées). En somme, 19 itinéraires photographiques sont mis en place entre 1994 et 2003 avec l'appui de l'État. Peu à peu, ce dernier se désengage financièrement de la démarche en ayant imposé a fortiori quelques contraintes et notamment le choix du photographe professionnel, un choix coûteux pour les institutions locales et une difficulté supplémentaire à investir le corpus à cause des droits d'auteur.

Toutefois l'OPNP a lancé « une véritable dynamique de la commande publique de photographies » (Bertho, 2009, p. 111) puisque depuis les années 2000 , on compte au moins $74^{6}$ Observatoires photographiques du paysage qui sont créés indépendamment du ministère de l'Environnement par des institutions locales (Pays, Parc Naturel Régional, Syndicat mixte, etc.) (figure 2 - planche XIII). Ici, l'État ne participe pas au financement des OPP. Cette autonomie permet à la gouvernance territoriale de faire des choix en adéquation avec le territoire et de s'approprier l'outil.

Les OPP dits locaux sont dispersés sur l'ensemble des régions françaises hormis la Haute-Normandie. L'expertise photographique qui a pour objet d'analyser les dynamiques paysagères est issue d'une longue tradition de la commande publique, mais aussi de la signature de la Convention européenne du paysage.

\section{L'expertise photographique : entre héritage de la commande publique et avènement du paysage ordinaire}

Un inventaire de démarches similaires aux OPP a été réalisé à l'international et 35 expériences ${ }^{7}$ peuvent être dénombrées en Europe, en Afrique,

6. Cet inventaire est non exhaustif et a été effectué en août 2015. Les OPP locaux ont été répertoriés sur internet, dans des articles scientifiques ou ils ont été présentés lors de communications.

7. Cet inventaire est non exhaustif et a été réalisé en 2016. en Amérique du Nord et du Sud (figure 3 planche XIV). Dans un premier temps, on peut constater que le protocole de la photographie répétée est largement employé et s'intègre essentiellement dans des projets de recherche pour l'étude de dynamiques paysagères spécifiques ou dans des projets individuels. À la différence de la démarche française, les points de vue initiaux sont rarement issus d'une concertation entre acteurs institutionnels et au regard des problématiques paysagères du territoire.

Trois types de démarches se dégagent:

- des Observatoires photographiques du paysage ou projets intégrant des reconductions photographiques comme Repeat photography in Bylot Island au Canada (Whitehead et al., 2010), Repeat Photography in Ethiopie (Nyssen et al., 2010) ou encore l'Observatorio y Archivo de los Paisajes de Andalucía (Jiménez, 2009).

- des Observatoires photographiques du paysage ou projets intégrant des reconductions photographiques avec une démarche participative dans laquelle les habitants sont invités à re-photographier des paysages comme l'Observatoire citoyen des paysages en Belgique ${ }^{8}$, Frisco city rephotography ${ }^{9}$ en Alabama.

- des Observatoires du paysage sans reconduction photographique où le but est de développer et de diffuser la connaissance paysagère tels que El Observatorio del Paisaje de Catalũna ${ }^{10}$, l'Osservatorio regionale per il paesaggio ${ }^{11}$ développé par la région de Vénétie.

Au final, l'échantillon international montre deux pôles principaux de développement des Observatoires. Le premier pôle correspond à l'Union européenne, l'engouement pour les préoccupations paysagères est dû à la Convention européenne du paysage $^{12}$ adoptée en 2000 par 29 pays membres. Ladite Convention promeut l'enrichissement des connaissances paysagères afin de développer des politiques d'aménagement, de protection et de gestion en adéquation avec les dynamiques paysagères. Le paysage ordinaire devient un enjeu sociétal et politique. Le second pôle correspond à l'Amérique du Nord qui fait écho à l'héritage des grandes expé-

8. À ce propos: [www.paysages-citoyens.be].

9. À ce propos: [friscocityrephotography.blogspot.com].

10. À ce propos : [http://www.catpaisatge.net].

11. A ce propos: [http://www.regione.veneto.it].

12. À ce propos : [http://www.coe.int/en/web/landscape]. 
ditions photographiques américaines de la fin du $\mathrm{XIX}^{\mathrm{e}}$ et début du XX $\mathrm{X}^{\mathrm{e}}$ siècle qui ont contribué à l'élaboration d'un fonds photographique considérable. Les instituts de recherche s'en sont emparés pour les re-photographier dans le temps.

La France reste une exception avec ses 98 OPP (OPP locaux et itinéraires de l'OPNP). En plus de la ratification de la Convention européenne du paysage en 2006, la commande photographique est une pratique institutionnelle courante en France depuis le XIX ${ }^{\mathrm{e}}$ siècle. Voici quelques exemples : la Mission héliographique de 1851, la Mission photographique de la Délégation interministérielle de l'aménagement du territoire et de l'attractivité territoriale (DATAR) dans les années 1980, la Mission photographique Transmanche de 1986-2006, la collection photographique du Conservatoire du littoral en cours de constitution depuis près de 30 ans, la collection de paysages autoroutiers (1986-1999) de la direction des Routes (Parcours photographique, 2015), etc. Dans ce sens, le fleurissement des OPP tient à la fois au contexte juridique français et à la place accordée à la culture visuelle dans les institutions. La nécessite de prendre en compte le paysage ordinaire dans les politiques sectorielles telles que celles du transport impose une compréhension des processus de transformation et d'évolution. Aussi, le suivi des travaux des infrastructures de transport est une thématique récurrente dans les Observatoires photographiques du paysage.

\section{Le suivi des infrastructures de transport : une thématique récurrente}

Comme le montre la figure 4 (planche XIV), les institutions qui portent un Observatoire sont essentiellement des institutions publiques dont plus d'un tiers sont en lien avec les politiques de protection des paysages (Parcs Naturels Régionaux, Labels Grand Site, Conservatoires ou Parcs Nationaux). Sur les 98 OPP inventoriés, 13 sont portés par des institutions moins classiques comme des laboratoires de recherche, des collèges ou encore des sociétés privées comme Eiffage Rail Express. Au-delà du caractère remarqué ou remarquable de ces paysages photographiés de par leur périmètre réglementaire, le paysage de l'ordinaire, subissant peu ou prou des bouleversements, fait également l'objet d'OPP. Les paysages d'entrées de ville, de zones industrielles, de friches urbaines sont dorénavant suivis. Dans le cadre des lois Grenelle, de la loi pour la reconquête de la biodiversité, de la nature et des paysages, les Observatoires deviennent aussi un instrument pour assister les schémas régionaux et locaux de planification comme l'OPP du Schéma Régional de Cohérence Écologique de la DREAL Bretagne (Legrand, 2013). Dans le même sens, l'OPP du Syndicat Mixte Provence Méditerranée accompagne le Schéma de COhérence Territoriale. Les Observatoires locaux sont des outils d'évaluation et d'aide à la prise de décision des politiques publiques (figure 5 - planche $\mathbf{X V}$ ).

Dans le cadre de la LGV Bretagne-Pays de la Loire, l'Observatoire a également une vocation d'évaluation mais aussi il a un véritable rôle dans la connaissance des paysages en cours de cicatrisation après rupture dans leur forme.

Le suivi photographique des grands projets d'infrastructure de transport représente $9 \%$ des OPP en France avec comme particularité d'observer les différentes phases de travaux : avant, pendant et après le projet. Chaque Observatoire a donc potentiellement une « fin de vie » en fonction de la cicatrisation escomptée des paysages.

Piloté par la DATAR Massif Central, l'Observatoire photographique des Territoires, débuté en 1999, s'attache à «montrer, évaluer, classifier, quantifier les différentes évolutions du paysage ${ }^{13}$ » suite à l'implantation de l'autoroute A89. Le CAUE de l'Orne s'intéresse à l'autoroute A26; la DREAL Nord-Pas-de-Calais, l'autoroute A1; le pays du Ruffécois la LGV Tours-Bordeaux, etc. L'expertise photographique des autoroutes est plus fréquente car les campagnes photographiques sont financées grâce au dispositif « $1 \%$ paysage, développement et cadre de vie » sur le réseau routier national. Issu de la circulaire du 31 mars 2005 et amendé par l'instruction gouvernementale du 29 juillet 2016 , le dispositif vise à «valoriser les paysages, remarquables et ordinaires, des collectivités territoriales concernées par un projet d'infrastructure routière ${ }^{14}$ ». La subvention de $1 \%$ du projet participe à différentes actions comme le traitement des entrées de ville, les aires de covoiturage, etc. en favorisant le développement économique et touristique des territoires.

13. Extrait du site internet : [www.esrifrance.fr/sig2006/blaise_pascal.html]. 14. Propos issu du site internet : [http://circulaire.legifrance.gouv.fr]. 
Par ailleurs, certains porteurs renouvellent la démarche OPP en intégrant les habitants dans la conception. Pour exemple, le Conseil général de Gironde met en place un OPP en partenariat avec les collèges de Saint-Yzan-de-Soudiac et de SaintAndré-de-Cubzac. Avec la collaboration de l'association « Passeurs », les collégiens sont sensibilisés aux mutations paysagères que provoque la LGV ToursBordeaux en leur demandant de réaliser et réitérer les photographies.

Cet état de l'art montre que l'OPP de la LGV Bretagne-Pays de la Loire s'intègre dans une dynamique d'expertise des infrastructures de transport au niveau national et contribue à la création d'une mémoire visuelle sur la cicatrisation des paysages. Parce que l'implantation de la LGV Bretagne-Pays de la Loire a des effets directs (remblais, déblais, protections acoustiques) et des effets indirects comme les opérations d'aménagement foncier (Guerrero et Pujols, 2011), la conception de l'OPP a été pensée en fonction de ces deux catégories d'impacts.

\section{SPÉCIFICITÉS DU SUIVI PHOTOGRA- PHiQue de LA LGV Bretagne-PAys DE LA LOIRE}

Les photographies qui composent l'OPP de la LGV Bretagne-Pays de la Loire sont réparties le long des $214 \mathrm{~km}$ de tracé. Elles exposent le cadre de vie de nombreux riverains et donnent à voir le paysage avant, pendant et après l'aménagement de la LGV. Les points suivants présentent les objectifs de l'OPP, sa méthode de conception et de reconduction des photographies. Le dernier point propose l'analyse évolutive de trois séries photographiques : (i) la LGV dans le paysage; (ii) les ouvrages de la LGV; (iii) la trame bocagère depuis la LGV.

\section{Les objectifs de l'OPP de la LGV Bretagne-Pays de la Loire}

Cet Observatoire photographique du paysage est élaboré à partir d'une concertation entre les experts ${ }^{15}$ du comité scientifique du volet environnemental de l'Observatoire socio-économique et environnemen-

15. Il est composé alors de membres d'Eiffage, de chercheurs de l'université Rennes 2 et de l'Institut de Recherche Agronomique de Rennes, d'un bureau d'assistante environnementale à maîtrise d'ouvrage, de l'association Bretagne Vivante et du Groupe d'Intérêt Public Bretagne Environnement. tal. ERE n'a pas mis en place un comité de pilotage spécifique à l'OPP. Le suivi photographique répond à la fois à des enjeux d'évaluation du projet en tant que tel à l'échelle locale, à des enjeux théoriques en matière de projets d'infrastructure et à des enjeux de partage de connaissance. Quatre objectifs se dessinent alors pour orienter l'OPP.

Le premier objectif vise à étudier l'intégration paysagère de la LGV au fil du temps en mesurant l'efficacité des mesures prises en faveur du paysage. Toutefois, l'analyse des photographies portera uniquement sur l'aspect visible de l'intégration de la LGV dans le paysage. En complément, le second objectif est d'observer les évolutions des paysages et de suivre leurs transformations, leurs altérations, leurs améliorations à partir de l'analyse comparative des photographies.

Ces deux points ont comme finalité d'exposer aux acteurs locaux les effets de la LGV sur leur territoire afin qu'ils infléchissent leurs activités ou leurs politiques. Ici, l'échelle locale est privilégiée, il s'agit d'intégrer - au-delà du "voir » - la LGV dans une perspective d'incorporation des nouvelles logiques bioécologique, foncière, sociale, économique, etc.

Troisièmement, l'Observatoire a comme but d'identifier et d'analyser les caractéristiques ainsi que les dynamiques et les pressions sur les paysages que modifie la LGV. Ce bilan a une dimension théorique puisqu'il sert à tirer des enseignements utiles en amont de projets nouveaux concernant la cicatrisation des paysages qui reste peu étudiée et en termes de résilience territoriale.

Le dernier objectif s'attache à partager la lecture des paysages auprès du plus grand nombre. Avec l'ouverture des données publiques - l'Open data-, rappelons que la LGV est le fruit d'un PPP, les données doivent être accessibles à tout un chacun en offrant des premières clefs d'analyse. Aussi, le partage des données fait sens avec les valeurs actuelles associées au paysage qui est considéré comme un «bien commun» (Sgard, 2010).

En réponse à cette triple ambition, la méthode a été conçue en adéquation avec la spécificité du projet et à partir d'un corpus déjà existant. 


\section{La méthode de conception et de reconduction photographique}

La mise en place de l'OPP repose en partie sur la méthodologie proposée par le ministère de l'Environnement (MEEDDAT, 2008). Néanmoins, il a été décidé d'ajuster la méthode compte tenu des objectifs visés et de l'échelle spatiale linéaire. La méthodologie adoptée présente donc des particularité, qui ne font pas pour autant de l'OPP de la LGV BPL un cas à part.

\section{Sélection des points de vue initiaux}

Ayant été acté après le démarrage des travaux de construction de la LGV, l'OPP est conçu à partir d'une couverture photographique préexistante réalisée par un bureau d'études d'aménagement paysager, l'atelier Pierre Lebrun, dans le cadre de l'étude d'Avant-Projet Sommaire (APS) entre 2003 et 2006. Ces photographies, issues d'une commande de SNCF Réseau, devaient constituer une référence visuelle des territoires, avant travaux. Près de 500 photographies, assemblées au format panoramique, ont ainsi été produites. Pour l'ensemble du corpus, un fichier informatique avait été constitué contenant le géoréférencement approximatif de chaque prise de vue avec son orientation et le nom du lieu-dit. L'atelier Pierre Lebrun a été impliqué dans l'ASP et dans le dossier d'enquête préalable à la Déclaration d'Utilité Publique (DUP). Déclarée d'utilité publique en 2007, l'État lance l'appel d'offre courant 2008. Le PPP est signé en 2011 avec un début des travaux fin 2012. Jusqu'au lancement de l'OPP en 2014, les photographies réalisées par le bureau d'étude n'ont pas été exploitées, elles n'avaient pas non plus pour dessein d'être reconduites dans le temps. Néanmoins, en 2014, elles offrent déjà un regard diachronique d'au moins 8 années. Le choix de prise de vue panoramique a pour effet d'embrasser la future LGV et ses abords paysagers. Au final, ERE n'a pas anticipé la création de l'OPP avant le commencement des travaux mais l'utilisation de cette archive documentée par des métadonnées permet alors de combler les lacunes. Nous pouvons constater qu'un tel dossier implique de nombreuses phases administratives et technocratiques, et de manière sous-jacente une multitude d'acteurs rendant le suivi complexe notamment dans l'exploitation des études préliminaires. C'est donc à partir des 500 clichés et deux ans après le début des travaux que le comité scientifique s’est réuni pour sélectionner les thématiques abordées par l'OPP au regard des photographies préexistantes (tableau 1).

Les deux thématiques « le réseau bocager » et « les espaces agricoles " sont symptomatiques des paysages traversés par la LGV et leur analyse apporte des clefs de lecture sur les opérations d'aménagement foncier. Quant aux «sites remarquables ou à enjeu ", ils permettent de s'intéresser à la covisibilité entre les paysages emblématiques du territoire à préserver et à la LGV. « La LGV avec et sans aménagements paysagers » portent sur les apports et les limites d'intégrer visuellement et de manière artificielle la voie ferrée (murs végétaux par exemple).

Par ailleurs, lors de la campagne photographique de l'Atelier Pierre Lebrun, le projet en n'était qu'à sa genèse, et donc certaines thématiques plus pointues, localisées et moins caractéristiques du paysage rural du Grand Ouest n'ont pu être développées qu'après concrétisation du projet. Une campagne complémentaire en 2014 est menée par la chargée de mission SIG pour imager les aménagements à vocation écologique (passages grande faune, mesures compensatoires) et pour suivre l'absorption des travaux (évolution des dépôts de matériaux).

Dans le même temps, la chargée de mission SIG s'est attachée à exclure certains clichés du corpus pour trois raisons (l'accessibilité, l'orientation et la distribution). Concernant l'accessibilité, les photographies doivent pouvoir être reconduites dans les mêmes conditions tous les ans et ce jusqu'à 2022, certains points de vue réalisés par l'Atelier Pierre Lebrun (2003-2006) et obstrués en 2014 ont donc été écartés du panel.

Outre cette condition pragmatique, la technicité photographique est prise en compte car elle conditionne «la géographie » de la photographie. En effet, le cliché doit donner à voir l'échelle paysagère adaptée au projet. Le point de vue est médian (Mendibil, 2008), il peut couvrir quelques centaines de mètres à quelques kilomètres afin d'observer les abords immédiats de la LGV et son contexte spatial pour les opérations d'aménagement foncier.

Aussi, la distribution géographique des photographies a été étudiée pour obtenir une couverture sur l'ensemble du tracé avec une répartition équilibrée. Les clichés sont des échantillons exemplaires de l'évolution du territoire impacté par la LGV. 


\begin{tabular}{|c|c|}
\hline THEME & DESCRIPTIF / PROBLEMATIQUE \\
\hline Le réseau bocager & $\begin{array}{l}\text { A observer: Alignement d'arbres, de haies et arbres isolés qui } \\
\text { forment une partie de la trame verte. } \\
\text { Nos interrogations: Est-ce que ces éléments s'étoffent ou au } \\
\text { contraire déclinent? }\end{array}$ \\
\hline Les espaces agricoles & $\begin{array}{l}\text { A observer : Occupation du sol. } \\
\text { Nos interrogations : Est-ce que nous observons aux abords de la } \\
\text { LGV un changement des pratiques agricoles? }\end{array}$ \\
\hline $\begin{array}{l}\text { La LGV avec et sans } \\
\text { aménagements } \\
\text { paysagers }\end{array}$ & $\begin{array}{l}\text { A observer : Intégration paysagère de la LGV } \\
\text { Nos interrogations : Les aménagements prévus pour dissimuler la } \\
\text { LGV sont-ils aussi efficaces que ce qui a été imaginé initialement? } \\
\text { Certains espaces font l'objet d'aménagements paysagers alors que } \\
\text { d'autres n'en ont pas, ces paysages gagnent-ils en qualité de } \\
\text { manière identique ou différente? } \\
\text { De même, l'aménagement étant localisé, crée-t-il une rupture avec } \\
\text { un espace à proximité qui n'en fait pas l'objet? La continuité } \\
\text { paysagère entre les deux existe-t-elle, est-elle inexistante ou se } \\
\text { créé-t-elle avec le temps? }\end{array}$ \\
\hline $\begin{array}{l}\text { Sites paysagers } \\
\text { remarquables ou à enjeu }\end{array}$ & $\begin{array}{l}\text { A observer: Plantations et merlons pour les paysages identifiées } \\
\text { comme à enjeux et remarquables. } \\
\text { Nos interrogations : L'attention particulière portée à l'ìnsertion de } \\
\text { sites traversés est-elle réussie? Perdent-ils de leur qualité } \\
\text { paysagère? S'ils la perdent, est-ce temporaire? Les } \\
\text { aménagements paysagers apportent-ils une plus-value par rapport } \\
\text { à avant? }\end{array}$ \\
\hline $\begin{array}{l}\text { Evolution des dépôts de } \\
\text { matériaux }\end{array}$ & $\begin{array}{l}\text { A observer: Intégration paysagère des dépôts. } \\
\text { Nos interrogations : Est-ce que le paysage spontané qui suit la fin } \\
\text { de la phase chantier est le même qu'avant travaux? La végétation } \\
\text { spontanée ou ensemencée fait-elle gagner en qualité le paysage } \\
\text { qui était différent avec les travaux? }\end{array}$ \\
\hline $\begin{array}{c}\text { Les passages à grande } \\
\text { faune (PGF) }\end{array}$ & $\begin{array}{l}\text { A observer: Développement de la végétation aux abords des } \\
\text { ouvrages. } \\
\text { Nos interrogations : Les plantations réalisées sont-elles suffisantes } \\
\text { pour rendre attractif les passages à grande faune? }\end{array}$ \\
\hline $\begin{array}{l}\text { La compensation } \\
\text { environnementale }\end{array}$ & $\begin{array}{l}\text { A observer: Evolution des milieux crées ou restaurés en faveur de } \\
\text { la biodiversité } \\
\text { Nos interrogations: Comment évoluent les aménagements } \\
\text { réalisés? }\end{array}$ \\
\hline
\end{tabular}

Tableau 1 : Thèmes abordés par l'OPP de la LGV Bretagne-Pays de la Loire Topics

addressed by the PLO for the Brittany - Pays de la Loire high-speed line (HSL)
Toutefois, ces clichés ne sont pas forcément représentatifs des dynamiques paysagères en œuvre, et donc cette équilibre reste illusoire en matière de représentativité des évolutions le long du tracé. Chaque point de vue nécessite une contextualisation spatiale plus large.

En somme, l'OPP est constitué de 82 points de vue initiaux répartis sur 35 communes de la Sarthe à l'Ille-et-Vilaine en passant par la Mayenne (figure 6 - planche XV).

\section{Reconduction des photographies}

Le principe de la série photographique est basé sur la fixité du cadrage de la prise de vue. Le point de vue initial délimite définitivement ce qui est dans le cadre et ce qui est hors cadre. Cette technique stricte et systématique facilite la comparaison des photographies entre elles. Concrètement, les campagnes photographiques respectent les quatre principes suivants :

- Les photographies sont prises au sol avec un trépied puisque les prises de vue doivent être les plus proches possibles de la perception optique de l'être humain afin de retranscrire au mieux les paysages ordinaires.

- Tant que possible, le lieu de prise de vue - là où le photographe se positionne dans l'espace pour prendre la photographie - se situe dans l'espace public pour éviter des potentiels conflits juridiques sur le droit de propriété. 
- Le pas de temps entre deux reconductions est régulier. Il peut toutefois évoluer au fil du temps s'il est justifié.

- Il est nécessaire de respecter des conditions similaires de prises de vue d'une reconduction à l'autre. Les conditions sont retranscrites dans des fiches pour faciliter le travail du photographe (date, heure, hauteur du trépied, distance focale, coordonnées GPS, etc.) Ces métadonnées peuvent aussi servir à l'analyse du corpus photographique, elles sont alors transférées dans une plateforme informatique accessible à tous, la POPP-Breizh (cf. supra). La première campagne de reconduction s'est déroulée de mars à octobre 2014. Les campagnes suivantes se sont déroulées sur la même période selon une fréquence annuelle. Les modifications du paysage étant moins brutales du fait de la fin des travaux de la $L G V$, cette fréquence a été révisée à la baisse en 2017. Les clichés sont dorénavant reconduits tous les deux ans. De plus, les personnes chargées de l'OPP ont changé de poste à la livraison de la LGV, les campagnes photographiques sont désormais réalisées par un autre chargé de mission. Les aléas liés au personnel avec des changements récurrents dans le suivi du dossier peuvent nuire à la cohérence de l'OPP si les informations n'ont pas été transmises correctement pour notamment reconduire les photographies mais aussi les analyser. Un Observatoire, pour qu'il soit efficient et permette une véritable évaluation, nécessite du temps et de la rigueur. Les chroniques photographiques ont déjà plus de dix ans et montrent des changements radicaux.

\section{Analyse de trois séries photographiques}

\section{La LGV au sein du paysage}

La première série photographique se situe en Ille-et-Vilaine sur la commune de Torcé. La photographie d'origine de 2005 s'inscrit dans un paysage bocager et de prairie (figure 7 - planche $\mathbf{X V I}$ ). Lors de la première campagne de reconduction en 2014, la prairie au premier plan est divisée en deux par les travaux de terrassement de l'infrastructure. Les remblais de la plateforme de la LGV sont bien visibles (figure 8 - planche $\mathbf{X V I}$ ). L'année suivante, la clôture de la ligne est en place et la végétalisation des talus contribue à intégrer la LGV dans le paysage. Les poteaux de signalisation et ceux de la caténaire sont en cours d'installation (figure 9 planche XVI). En 2016, les poteaux caténaires sont bien en place avec les fils de contact, la voie ferrée est implantée. La présence des moutons témoigne de la reprise de l'activité agricole (figure $10-$ planche XVI). Cette série photographique aborde trois thématiques : «le réseau bocager »; «les espaces agricoles » et « la LGV avec et sans aménagements paysagers ». Le point de vue est orienté vers la LGV, il montre au final de nouveaux marqueurs dans le paysage (poteaux) et une résilience en matière d'aménagement foncier.

\section{Les ouvrages de la LGV}

La photographie d'avril 2004 est prise depuis un chemin de halage en bordure de la Mayenne à Changé. Des maisons se trouvent au bord de l'eau sur la rive droite et en arrière-plan se devine le pont de l'autoroute A81. Sur la rive gauche, à la lisière du chemin, un terrain de golf avec sa pelouse et un des plans d'eau du parcours sont visibles (figure 11 - planche XVII). En 2014, le viaduc sur la Mayenne est déjà implanté sur ses culées en remblai (figure 12 - planche XVII). En 2015, les réaménagements prévus au pied du viaduc ont eu pour conséquence la destruction d'une partie des maisons et des arbres situés sur la rive droite. Un projet de réaménagement du golf de Changé a été mis en œuvre et l'augmentation de surface de la pièce d'eau visible sur les photographies en est une conséquence (figure 13 - planche XVII). En 2016, la présence des carrés de feutre de chaque côté du viaduc traduit l'effort de plantation réalisé pour renforcer l'intégration paysagère du viaduc (figure 14 - planche XVII). La chronique photographique peut s'apparenter à deux thèmes : « la LGV avec et sans aménagements » avec cette volonté de verdir les remblais et « les sites paysagers remarquables ou à enjeu ». En définitive, l'ampleur du viaduc casse la linéarité des éléments paysagers caractéristiques de la Mayenne, à savoir le chemin de halage et son cours d'eau. Ici encore, le point de vue est orienté vers la LGV.

\section{La trame bocagère depuis la LGV}

Dernier exemple en Sarthe dans la commune de Coulans-sur-Gée. Contrairement aux séries photogra- 
phiques présentées précédemment, celle-ci tourne le dos à la ligne. Elle a pour objectif d'observer le réaménagement foncier suite à l'implantation de la LGV, le point de vue est depuis la LGV. La photographie du mois de juin 2003 expose une vue en arrière-plan sur le château du village masqué en partie par des arbres ornementaux. Les façades et toitures sont inscrites au titre des monuments historiques. Le premier plan est occupé par une prairie puis des champs cultivés avec quelques haies, un poteau électrique et une vieille maison (figure 15 - planche XVIII). Peu de changement en 2014, la prairie du premier plan est en culture, les limites de ce champ marquées par la haie sont davantage soulignées. La maison a également été restaurée et un jardin a été aménagé (figure 16 planche XVIII). Juin 2015, les haies situées au premier plan disparaissent. Cette suppression résulte du regroupement parcellaire lié à l'aménagement foncier. En revanche les haies situées dans le périmètre de protection du château sont préservées. Le jardin d'ornement de la maison s'étoffe. Le poteau électrique est toujours présent (figure 17 - planche XVIII). La dernière photographie ne présente pas de changement significatif (figure 18 - planche XIX). Cette série photographique est assimilée aux deux thématiques agricoles et aux « sites paysagers remarquables ou à enjeu ». La disparition progressive des éléments de paysage et l'uniformisation des cultures ont banalisé le paysage photographié.

L'Observatoire photographique du paysage permet de restituer pas à pas les changements paysagers depuis la LGV ou vers la LGV. Les micro-changements donnent des indices sur les évolutions des paysages, et indirectement sur les recompositions territoriales en particularité par rapport à l'aménagement foncier. Toutefois, pour valider les analyses, il est essentiel de réaliser un contrôle de terrain, d'autant plus que ces dynamiques ne sont pas forcément représentatives de l'ensemble du tracé. Une contextualisation spatiale doit ainsi être engagée pour affiner les études, et c'est pour cela que ERE s'est inséré dans le réseau régional des OPP de Bretagne.

\section{L'OPP de la LGV Bretagne-Pays DE LA LOIRE : DE L'ÉCHELLE DU PROJET À L'ÉCHELLE RÉGIONALE}

La diversité des thématiques suivies et les spécificités du médium photographique permettent de relier les problématiques traitées par l'Observatoire environnemental. Aussi, l'implication d'ERE dans le réseau breton des OPP donne lieu à un partage des données visuelles à l'échelle régionale. Ainsi, les séries photographiques sont accessibles à tous et peuvent être analysées avec d'autres corpus OPP. Les dynamiques paysagères peuvent être analysées à différentes échelles : de l'échelle locale à l'échelle régionale.

\section{De la série photographique au bilan LOTI}

Aujourd'hui, le corpus photographique est en cours de constitution avec la réalisation de quatre campagnes photographiques depuis 2014. Les transformations visibles sont notables et donnent à voir la phase de synchronie (partie 2). Les reconductions suivantes ont pour objectif d'analyser la résilience des paysages en mettant également en lumière les dynamiques saisonnières (cycle végétal, déplacement de la faune). Comme le montre la figure 19, l'exploitation future pourrait servir à un bilan multidimensionnel dans le cadre de l'Observatoire environnemental. Les micro-changements visibles sur les séries photographiques permettent de confirmer et d'illustrer les tendances observées dans les différentes branches du volet environnement. Aujourd'hui, nous avons peu d'éléments sur la faisabilité de ce bilan potentiel par ERE.

\section{Évaluation du Schéma Directeur Paysager}

L'analyse pourrait servir à évaluer le Schéma Directeur Paysager réalisé dans le cadre de l'Avantprojet détaillé (ERE, 2011). Après une étude des quatre entités relatives au tronçon de la LGV (le bassin rennais, la vallée de la Mayenne, les affluents de la Sarthe, les alentours du Mans), il définit le projet d'aménagement pour chaque zone en réponse à trois grands principes.

Premièrement, il s'agit de "maintenir l'identité de ce paysage de bocage aux abords de la ligne » (ERE, 2011, p. 9). La série photographique de Torcé (figures 7 à 10 - planche XVI) illustre cette identité bocagère. Ce principe s'apparente à une volonté de préserver une caractéristique régionale (Margetic et al., 2014), il reste assez illusoire en matière de fonctionnement écologique des réseaux bocagers. 
Figure 19 : Transversalité de l'OPP au sein de l'Observatoire environnemental Transversal nature of the PLO within the Environmental Observatory

\section{Observatoire environnemental}

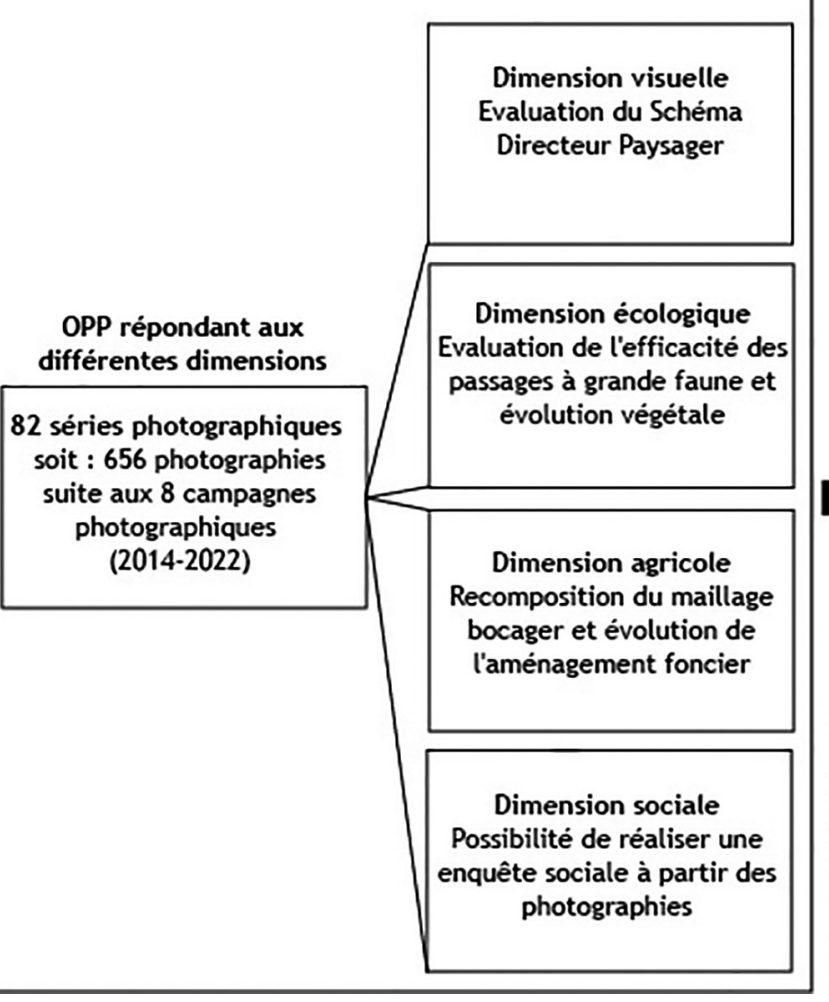

Néanmoins, l'OPP donne déjà des clefs de compréhension pour traiter ce point.

Deuxièmement, le projet d'aménagement précise de « prévoir les épaisseurs d'emprises nécessaires à ces modelés et ces plantations » (ERE, 2011, p. 9). Pour ce faire, deux traitements morphologiques sont envisagés et dessinés : " modeler les abords de la ligne de façon à masquer le projet depuis l'habitat riverain; prévoir les épaisseurs d'emprises nécessaires à ces modelés et ces plantations » (ERE, 2011, p. 9).

Les séries photographiques sont à une échelle micro montrant ces modelés et ces plantations. Les prises de vue vers la LGV participent à évaluer ce principe. Pour compléter l'évaluation, une enquête auprès des riverains serait nécessaire afin de mesurer la perception habitante sur le masquage visuel de la voie ferrée.

De plus, les trames végétales rompues peuvent être complétées par des boisements, arbres isolés ou vergers. La série photographique de Torcé-Mauzé image ces prescriptions avec la création d'un remblai et de sa végétalisation pour amenuiser l'impact visuel de la LGV. Dans certains cas, l'Observatoire photographique du paysage présente des paysages en voie de cicatrisation où l'évaluation de l'aménagement nécessite du temps. La série la Juquelière à Saint-Cyr-le-Gravelais (53) témoigne de ce constat (figures 20 et 21 - planche XIX). Malgré les plantations réalisées en 2016 (à droite de la figure 21 - planche XIX), le caractère bocager du lieu a été considérablement fragilisé. L'OPP permet ici, par l'étude de cas concrets exemplaires - potentiellement 82 cas puisque 82 points de vue - d'évaluer la conformité des grands principes dictés par le Schéma Directeur Paysager.

Toutefois, le Schéma Directeur Paysager est un document aux fondements critiquables puisqu'il prend en compte essentiellement la dimension visuelle des paysages. De façon caricaturale, les prescriptions ont pour objectif de " camoufler » la LGV dans une perceptive de paysage-masque ou de créer des perspectives visuelles pour l'usager de la LGV dans une optique de paysage-décor. La dimension écologique du paysage, comme par exemple le rôle écologique de l'implantation de nouvelles haies, n'est pas considérée dans le projet d'aménagement 
du Schéma Directeur Paysager. Dans le même sens, l'impact sonore de la LGV ne participe pas à l'évaluation du projet, alors que les conséquences du bruit des transports sur la santé mentale, mais aussi sur la faune et la flore, sont aujourd'hui démontrées (Vincent et Olny, 2012). Le paysage se rapporte ici à une conception esthétisante héritée de la culture visuelle (Guittet, 2016). La qualité paysagère, incluant le bon fonctionnement écologique, la qualité sonore, la qualité du cadre de vie perçue comme agréable par les habitants, n'est pas considérée. L'analyse des dynamiques paysagères est donc ici relative à l'échelle locale et se réalise par la comparaison d'éléments de paysage ${ }^{16}$ visibles sur les photographies traduisant la modification de la structure paysagère ${ }^{17}$.

\section{L'OPP en réponse aux axes de l'Observatoire environnemental}

Comme le montre la figure 16 (planche XVIII), l'OPP est un appui analytique pour enrichir d'autres dimensions de l'Observatoire environnemental. Premièrement, les séries photographiques peuvent être une ressource supplémentaire pour analyser les dynamiques écologiques. En effet, les séries photographiques consacrées aux passages à grande faune et à la compensation environnementale apportent des indicateurs sur les potentielles conquêtes végétales, des indicateurs in fine sur la biodiversité floristique. Ce type d'expertise est d'ailleurs récurrente et probante aux États-Unis (Webb et al., 2010).

Deuxièmement, les trois quarts des images du corpus présentent des paysages agricoles où l'impact indirect de la LGV peut être étudié au regard de l'aménagement foncier et des éventuelles modifications de pratiques agraires. Reprenons l'exemple de la série photographique de Torcé (35), où l'élevage ovin perdure malgré la division parcellaire (figures 4 à 7 - planches XIV-XVI). Concernant les photographies de Coulans-sur-Gée (72), l’implantation de l'infrastructure a entraîné un regroupement parcellaire où l'on passe d'une polyculture céréa-

16. Les éléments de paysage «participent aux structures paysagères (exemple : la haie composée de pruneliers dans certains paysages de bocage du nord-ouest de la France » (MEDDE, 2015, p. 30). Ils sont révélateurs des caractéristiques paysagères plus étendues.

17. Les éléments interagissent de manière systématique, se dessinent alors des structures paysagères. Ces dernières ont trois dimensions : « topographiques, fonctionnelles et symboliques » (MEDDE,2015, p. 30). lière à une monoculture céréalière (figures 15 à 18 - planche XVIII-XIX). Ce cas n'est pas isolé, d'autres séries photographiques donnent à voir une évolution des pratiques : de l'élevage à la monoculture. L'analyse approfondie des changements de pratiques pourrait être couplée avec la recomposition du maillage bocager et de son entretien.

Troisièmement, en écho avec les enquêtes psychosociologiques menées par le bureau Egis concernant les perceptions de la LGV par les riverains vivant à proximité $^{18}$, l'Observatoire photographique du paysage pourra être le support de la prochaine enquête sociale prévue au tournant des années 2020 afin de souligner les évolutions de perceptions des riverains. Avec la méthode de la photo elicitation interview (Harper, 2002; Bigando, 2013; Guittet, 2013), les changements visibles contenus dans les séries photographiques pourront être commentés, appréciés ou dépréciés donnant alors des indicateurs concernant l'acceptabilité sociale sur l'intégration de la LGV dans le paysage et plus largement dans le quotidien des habitants (Guittet, 2016).

Enfin, l'interprétation des photographies alimentera les données nécessaires au bilan LOTI. En réponse à la Loi d'Orientations des Transports Intérieurs (LOTI) de 1982, ce bilan doit être réalisé trois et cinq ans après la mise en service des infrastructures. Il comporte un volet socio-économique et un volet environnemental dans lequel s'inscrira le paysage.

L'intérêt de l'analyse paysagère tient à sa transversalité, notamment dans la compréhension des mutations territoriales et dans l'appréhension des représentations des populations. L'OPP peut un support pour développer des indicateurs sur les dynamiques éco-biologiques et territoriales mais aussi sur la dimension sociale (des perceptions individuelles aux représentations collectives, des usages aux pratiques).

18. 526 riverains ont été interrogés sous forme de questionnaires, d'entretiens semi-directifs et d'entretiens libres dans huit communes concernées par l'implantation de la LGV - Cesson-Sévigné, Domagné, Brielles, Changé, La Cropte, Poilé-sur-Vègre, Coulans-sur-Gée, Secteur périurbain entre Aigné et Maquère -. Ces enquêtes sociales ont été menées par les étudiants du MASTER Aménagement et Collectivité Territoriale de l'université Rennes 2 (2013-2014) et du MASTER Biodiversité Territoire et Environnement de l'université Paris 1 (2014-2015). 


\section{De l'échelle de la LGV à l'échelle régionale}

Dès la création de son Observatoire photographique du paysage en 2013, Eiffage Rail Express participe à un réseau d'OPP bretons qui a pour origine le développement d'une plateforme informatique commune : la POPP-Breizh ${ }^{19}$. Piloté par le Conseil régional de Bretagne, la Direction Régionale de l'Environnement, de l'Aménagement et du Logement (DREAL) de Bretagne et par le laboratoire ESO-Rennes, ce projet lancé en 2011 a pour objectif de rendre accessible et exploitable les OPP bretons en mutualisant les moyens et les compétences des porteurs (Le Dû-Blayo et Guittet, 2016).

\section{L'exploitation de l'OPP de la LGV Bretagne- Pays de la Loire}

Sans recours à l'outil informatique, l'accumulation des données photographiques (et des métadonnées associées aux prises de vue) rend difficile l'analyse des dynamiques paysagères. Un traitement semiautomatique est implémenté dans la POPP-Breizh pour faciliter l'exploitation des données. Il vise à synthétiser les changements qui affectent chaque élément de paysage ${ }^{20}$ repéré sur les séries photographiques par un code qui simplifie les dynamiques (stabilité, apparition, disparition, augmentation, diminution, changement d'aspect). Ce traitement est rendu possible grâce à la conception d'un thésaurus composé d'éléments de paysage ${ }^{21}$. ERE a ainsi conçu un thésaurus spécifique aux problématiques liées à la LGV qui est également imtégré (exemple de termes : avifaune, mur anti-bruit, etc.).

Les données statistiques issues de ce travail apportent alors un aperçu complet des éléments de paysage visibles dans le corpus et de leurs mutations. Ainsi, l'analyse des dynamiques paysagères est facilitée pour appréhender l'évolution des éléments et des structures de paysage. Par le traitement semiautomatique, l'OPP de LGV peut être traité avec d'autres corpus OPP. En effet, d'autres points de

19. Plateforme des Observatoires Photographiques du Paysage de Bretagne. En ligne depuis 2015, elle est accessible à l'adresse suivante : [https:// popp.applis-bretagne.fr/].

20. Depuis la loi «paysage » de 1993, le terme d'élément de paysage a une signification partagée dans les politiques publiques en France.

21. Ce dernier a été élaboré à partir du contenu visuel des séries photographiques bretonnes puis il a été homogénéisé avec d'autres thésaurus disponibles. Enfin, il a été testé par un échantillon d'acteurs du territoire. vue peuvent compléter le corpus d'ERE et aider à contextualiser plus largement les impacts de la LGV.

En plus de la mise à disposition des séries photographiques auprès du grand public (élus locaux, habitants, associations naturalistes, etc.), la POPPBreizh permet aux différents membres du comité de pilotage de l'Observatoire environnemental de s'approprier les photographies en les recherchant par exemple par commune, par mot clef et/ou par dynamique « je recherche toutes les séries photographiques où des haies ont disparu » - et d'aider dans la rédaction du bilan LOTI. La plateforme relie les thématiques de l'Observatoire environnemental, rend exploitable le corpus par tous et donne à voir concrètement les impacts de la Ligne à Grande Vitesse - au-delà des chiffres et autres indicateurs. Visualiser les impacts, c'est rendre concret les actions des acteurs.

\section{Un partage de connaissance régional}

Au-delà de ses propres intérêts pour l'exploitation du corpus via la POPP-Breizh, ERE contribue à une synergie entre acteurs du territoire qui participent de près ou de loin aux transformations paysagères. Le projet de la plateforme informatique réunit aujourd'hui une quarantaine d'institutions et quinzaine de porteurs d'OPP bretons. Cette collaboration a un triple enjeu : (i) discuter et débattre des évolutions paysagères; (ii) partager les innovations techniques et méthodologiques OPP; (iii) partager les données photographiques pour enrichir la connaissance paysagère à l'échelle régionale. Les données contenues dans la plateforme informatique fourniront à terme des clefs de lecture sur les dynamiques paysagères régionales.

De surcroît, étant en Open Source, la POPP peut être installée au sein des serveurs des autres collectivités territoriales ou des réseaux d'acteurs, avec comme ambition d'analyser les changements paysagers à l'échelle interrégionale en rendant les interfaces interopérables ${ }^{22}$ (figure 22 - planche $\mathbf{X X}$ ). Étant donné que ERE a développé un thesaurus spécifique aux impacts de la LGV, la plateforme peut devenir un moyen de mieux cerner les enjeux

22. « L'interopérabilité est la capacité que possède un système, dont les interfaces sont intégralement connues, à fonctionner avec d'autres systèmes existants ou futurs sans restriction d'accès ou de mise en œuvre. » (Roberts, 2015, p. 370). 
de la cicatrisation des paysages si d'autres projets d'infrastructures se développent.

Eiffage Rail Express concourt au développement d'un outil de coproduction des savoirs paysagers : coproduction par l'implication de différents porteurs OPP (acteurs du secteur public, acteurs du secteur privé, particuliers, etc.) et coproduction de savoirs hétérogènes par l'investissement potentiel des habitants via des commentaires en ligne, des enregistrements sonores, etc.

\section{Conclusion}

Souvent réduit à son rôle illustratif (Dérioz et al., 2010), l'OPP est pourtant un outil qui enregistre en continu - tous les ans, tous les deux ans, tous les dix ans - les mutations territoriales en réhabilitant la vue horizontale, " une partie de territoire telle que perçue par les populations » (Convention européenne du paysage, 2000). L'impact des grandes infrastructures de transport sur l'évolution des paysages ordinaires est dorénavant évalué. L'analyse visuelle et statistique des séries photographiques par le biais de la POPP-Breizh alimente les différents volets traités dans le cadre de l'Observatoire environnemental et mais aussi le bilan LOTI. Cette analyse est complémentaire avec des études de télédétection et du travail de terrain. L'analyse du corpus photographique n'est pas suffisante car les points de vue ne sont pas forcément représentatifs des processus de cicatrisation et l'analyse ne peut se réduire à l'approche visuelle. Le paysage est aussi sonore, a une dimension sociale et participe à un fonctionnement plus complexe en termes bioécologique et territorial. L'OPP donne à voir concrètement l'impact du projet sur le territoire, il montre aux acteurs impliqués les effets de leurs prises de décision. Pour exemple, le réaménagement foncier et les micro-changements qu'il engendre dans les paysages sont observables alors qu'il s'agit de processus discrets, presque invisibles. Pourtant « petite touche par petite touche ", le réaménagement foncier peut entraîner une banalisation des paysages, une perte de l'identité régionale. La chronique photographique rend alors visible l'invisible.

La veille photographie impose a fortiori une veille biannuelle in situ, ce qui permet à Eiffage Rail
Express de rester au plus près du terrain. Toutefois, la livraison de la LGV en 2017 a provoqué des mouvements de personnel, ainsi le suivi photographique a été délégué à un autre chargé de mission qui doit se familiariser avec le corpus photographique et le terrain. Après quatre ans d'OPP, ERE a réalisé un premier bilan de l'outil. D'un part, il est constaté que certaines thématiques paysagères sont sous-représentées dans le corpus (sites paysagers emblématiques, zones de dépôts), et d'autre part, l'enquête psychosociologique (2013-2015) aurait pu être menée en lien avec l'OPP. La participation des habitants dans la démarche OPP (prises de vue, captations sonores avant/pendant/après les travaux, discussions à partir des séries photographiques) aurait pu permettre une meilleure compréhension de l'évolution des représentations individuelles et sociales des riverains face à l'implantation de la LGV, et contribuer à une mémoire sociale des mutations paysagères liées au projet.

On l'a vu, l'OPP de la LGV Bretagne-Pays de la Loire est intégré dans un projet régional voire interrégional sur la mise en connaissance des dynamiques paysagères. Eiffage Rail Express a en charge l'Observatoire environnemental pour 10 ans selon la Charte avec SNCF Réseau. Néanmoins, le suivi photographique de certains paysages nécessiterait d'être prolongé au-delà de 2022 afin d'observer dans le temps les processus de cicatrisation. C'est un exemple de plus qui montre, que malgré une volonté d'ERE de contribuer à l'élaboration du savoir, le décalage temporel entre le temps des paysages et le temps des acteurs du territoire persiste, un décalage causé notamment par les procédures technocratiques.

Les Observatoires photographiques du paysage semblent faire le trait d'union entre le paysage politique et le paysage vernaculaire. Le paysage politique est symptomatique des manières d'agir et de marquer les paysages à l'échelle régionale. Le paysage vernaculaire est l'expression des manières d'habiter et d'être dans le paysage à l'échelle locale. Les Observatoires retranscrivent les deux. C'est en cela qu'ils peuvent être partagés entre les politiques publiques, la sphère privée et les citoyens. 


\section{Bibliographie}

Aschan-Leygonie C., 2000. Vers une analyse de la résilience des systèmes spatiaux, L'espace géographique, Tome 29, n 1 , p. 64-77.

Bigando E., 2013. De l'usage de la photo elicitation interview pour appréhender les paysages du quotidien : retour sur une méthode productrice d'une réflexivité habitante, Cybergeo: European Journal of Geography, [http://cybergeo.revues. org/25919].

Bertho R., 2009. « Du territoire au paysage, la Mission photographique de la DATAR et l'Observatoire photographique du paysage ", in Actes de la journée d'étude Photographier le territoire, Paris, Somogy, p. 109-117.

Bailly J.-C. (dir.), 2013, Les cicatrices du paysage, Cahier de l'École de Blois, La Villette, Paris, 96 p.

Carré J., Métailié J.-P., 2008. De los paisajes de ayer a los paisajes de mañana. Metodología de un observatorio fotográfico para el análisis de las dinámicas paisajísticas: el valle de Vicdessos, Pirineos de Ariége (Francia), Cuadernos geográficos, n 43, p. 123-149.

Chouquer G., 2000. L'étude des paysages, essais sur leurs formes et leur histoire, Paris, Errance, 208 p.

Dérioz P., Béringuier P., Laques A.-E., 2010. Mobiliser le paysage pour observer les territoires : quelles démarches, pour quelle participation des acteurs?, Développement durable et territoires, Vol. 1, no 2. http://developpementdurable.revues.org/8682.

Eiffage Rail Express, 2011, Schéma Directeur Paysager, 216 p.

Guerrero A., Pujols J.-P., 2011. Le paysage dans la conception et la réalisation des lignes nouvelles à grande vitesse, Revue d'histoire des chemins de fer, $\mathrm{n}^{\circ}$ 32-33, [http://rhcf. revues.org/581].

Harper D., 2002, Talking about pictures: a case for photo elecitation, Visual Studies, Vol. 17, n 1, p. 13-26.

Guittet C., 2016. Pour une meilleure intégration des Observatoires Photographiques du Paysage dans la gowvernance territoriale (exemple de la région Bretagne), Rennes, Université Rennes 2, 836 p. [https://www.theses.fr/197171788].

Guittet C., 2013. Quand les habitants examinent les dynamiques paysagères : l'utilisation de photographie dans les entretiens de recherche, ESO, Travaux et documents, $\mathrm{n}^{\circ} 36$, p. 109-115.

Jiménez Olivencia Y. (dir.), 2009. Memoria final del proyecto de Investigación: «Observatorio y Archivo de los Paisajes de Andalucía » (OAPA), Rapport de recherche, Granada, Universidad de Granada, 469 p.

Le Dû-Blayo L., Guittet C., 2016. Un projet partagé au service des acteurs : la plateforme des Observatoires photographiques du paysage en Bretagne (POPP-Breizh), Projet de paysage. [http://www.projetsdepaysage.fr/un_projet_partag_ au_service_des_acteurs_la_plateforme_d_observatoires_ photographiques_du_paysage_en_bretagne_popp_breizh_]. Lebart L., 2002. « La "restauration” des montagnes », Études photographiques, n. 3, [http://etudesphotographiques. revues.org/96].

Legrand H., 2013, L'Observatoire Photographique des paysages de la trame verte et bleue de Bretagne: outil de suivi et d'évaluation "sensible » du schéma régional de cohérence écologique, Rapport de stage de Master 1 Gestion Territoriale du Développement Durable, Université Michel-Montaigne Bordeaux 3, 63 p.

Margetic C., Bermond M., Jousseaume V., Marie M. (coord.), 2014. Atlas des campagnes de l'Ouest, Rennes, Presses universitaires de Rennes, 304 p.

MEDDAT, 2008. Itinéraires photographiques: Méthodes de l'Observatoire photographique du paysage, Paris, MEEDDAT, $74 \mathrm{p}$.

MEDDE, 2015. Les Atlas de paysage. Méthode pour l'identification, la caractérisation et la qualification des paysages, Paris, MEDDE, $115 \mathrm{p}$.

Mendibil D., 2008. Dispositif, format, posture : une méthode d'analyse de l'iconographie géographique, Cybergeo: European Journal of Geography, [http://journals.openedition.org/ cybergeo/16823; DOI : 10.4000/cybergeo.16823].

Nyssen J., Frankl A., Munro R.N., 2010. Repeat Photography in Éthiopie: Digital Photographic Archives for Environmental and Historical Studies: An Example from Ethiopia, Scottish Geographical Journal, Vol. 126, n 3, p. 185-207.

«Parcours photographique », 2015. Numéro thématique de Pour mémoire, comité d'histoire, dirigé par R. Bertho, n 16, $168 \mathrm{p}$.

Roberts R, 2015. Les archives en 2030. De la donnée (signifiant) à l'information sur la donnée (signifié) au service des documentalistes et autres publics, in Servais Paul (dir.), Mirguet Françoise (collab.), Archivistes de 2030. Réflexions prospectives, Louvain-la-Neuve, L'Harmattan, p. 369-394.

Vincent B., Olny X., 2012. Le bruit des transports, une nuisance, un impact majeur pour la population, Pollution atmosphérique, $\mathrm{n}^{0} 215$, p. 225-236.

Webb H.R., Boyer E.D., Turner M.R., 2010. Repeat Photography, Methods and Applications in the Natural Sciences, Washington, IslandPress, $392 \mathrm{p}$.

Whitehead K., Moorman B., Wainstein P., Habib A., 2010. Monitoring Rapidly Evolving Landscape Features Using Ground-Based Time-Lapse Photography: A Case Study for a Proglacial Icing, Canadian Geomatics Conference, Calgary, Alberta, s.p. [http://www.isprs.org/proceedings/XXXVIII/ part1/02/02_03_Paper_67.pdf]. 